\title{
Erratum to: Highlighting consensus among medical scientists increases public support for vaccines: evidence from a randomized experiment
}

\author{
Sander L. van der Linden ${ }^{1 *}$, Chris E. Clarke ${ }^{2}$ and Edward W. Maibach ${ }^{2}$
}

\section{Erratum}

Following the publication of this article [1], it was brought to our attention that Table 2 of the Appendix contains a typographical error. The right column of the table incorrectly reads $(N=216)$ instead of $(N=206)$.

In addition, Table 3 of the Appendix contains some non-consequential rounding errors for the Mean (S.D.). The corrected table is provided below:

The above errors do not influence the findings and conclusions presented in the article [1].

\section{Author details}

'Department of Psychology and Woodrow Wilson School of Public Affairs, Princeton University, Princeton, NJ, USA. ${ }^{2}$ Department of Communication, George Mason University, Fairfax, VA, USA.

Received: 22 March 2017 Accepted: 22 March 2017

Published online: 29 March 2017

\section{Reference}

1. van der Linden, et al. SWITCH: Highlighting consensus among medical scientists increases public support for vaccines: evidence from a randomized experiment. BMC Public Health. 2015;15:1207. doi:10.1186/ s12889-015-2541-4.

\footnotetext{
* Correspondence: sander.vanderlinden@princeton.edu

'Department of Psychology and Woodrow Wilson School of Public Affairs, Princeton University, Princeton, NJ, USA
} 
Table 3 Survey questions and descriptive statistics

Sample

Mean (S.D.)

Survey questions

Perceived scientific agreement

To the best of your knowledge, what \% of medical scientists agree that vaccines are safe? $(0 \%-100 \%)$

$88.57(10.14)$

Autism-vaccine link

To what extent do you agree with the following statement; "there is scientific evidence for a causal link between vaccines and autism" (1 = Completely Disagree $-7=$ Completely Agree)

Risk perception/concern

How concerned are you about the potential risk of vaccines? ( 1 = I am not concerned at all, $7=\mathrm{I}$ am very concerned).

Public support index (strongly disagree $=1$, strongly agree $=7$ ).

I believe that vaccines are a safe and reliable way to avoid the spread of otherwise preventable diseases $(M=6.29, S D=1.20)$

I have already vaccinated my children or would do so if I had children $(M=6.29, S D=1.52)$.

I would support policies that require people to vaccinate their children $(M=5.73, S D=1.78)$.

I believe that the health benefits of vaccines outweigh the risk of any potential negative side effects $(M=6.16, S D=1.38)$.

I believe that vaccines are important in maintaining and improving public health $(M=6.31, S D=1.25)$.

In the interest of public health, parents should simply be required to vaccinate their children $(M=5.76, S D=1.70)$

More people ought to vaccinate themselves and their children $(M=6.21, S D=1.48)$.

I believe that vaccine refusal poses a risk to public health $(M=6.0, S D=1.62)$ 